\title{
Drittmitteleinwerbung Jeder Antrag zählt
}

\author{
Heike Faßbender und Volker Mehrmann
}

Durch Drittmittel unterstützte Forschung hat sich zu einem zentralen Element in der (deutschen) Forschungslandschaft entwickelt. Die Drittmitteleinwerbung ist an vielen Hochschulen ein wichtiges Ziel und das Engagement bzw. der Erfolg der Drittmitteleinwerbung ein Kriterium für Zulagen beim persönlichen Gehalt und Zusagen an Ausstattung durch die jeweilige Hochschulleitung. Unser Anliegen in diesem kurzen Beitrag ist die Sensibilisierung, dass sich bei einigen für die Mathematik besonders relevanten Drittmittelgebern wie der Deutschen Forschungsgemeinschaft (DFG) oder dem European Research Council (ERC) die Höhe des Verfügungsrahmens für die einzelnen Fächer aus dem relativen Antrags- und Bewilligungsvolumen der Fächer bezogen auf die gesamte Antrags- und Bewilligungssummen über alle Fächer hinweg berechnet.

Bezogen auf die DFG schneidet die Mathematik bei dieser Aufteilung der Fördermittel gut ab. In den Jahren 2015 bis 2018 ist der DFG-Fördertopf Mathematik von 68,8 Mio€ auf 89,9 Mio€ gewachsen, siehe Abbildung 1. Dies ist im Vergleich zu anderen naturwissenschaftlichen Fächern eine erfreuliche Steigerung. Bei der Analyse der Zahlen ist zu beachten, dass es neben den aus dem DFG-Fördertopf der Mathematik finanzierten Projekten auch komplett aus anderen DFG-Fördertöpfen geförderte Projekte aus der Mathematik gibt. Solche Projekte/Bewilligungen sind in den folgenden Abbildungen nicht erfasst.

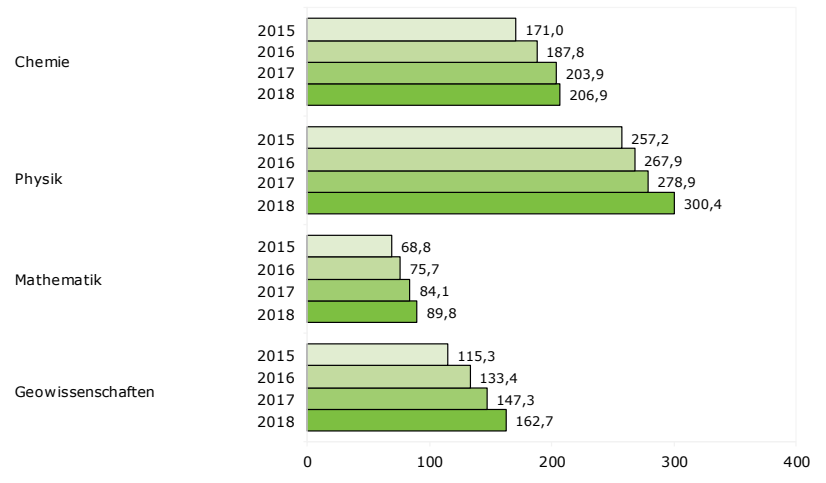

Abbildung 1. Jahresbezogene Bewilligungen für laufende Projekte je Fachgebiet 2015 bis 2018 (in Mio. $€$ ) in den Naturwissenschaften (Quelle: DFG-Jahresbericht 2018)

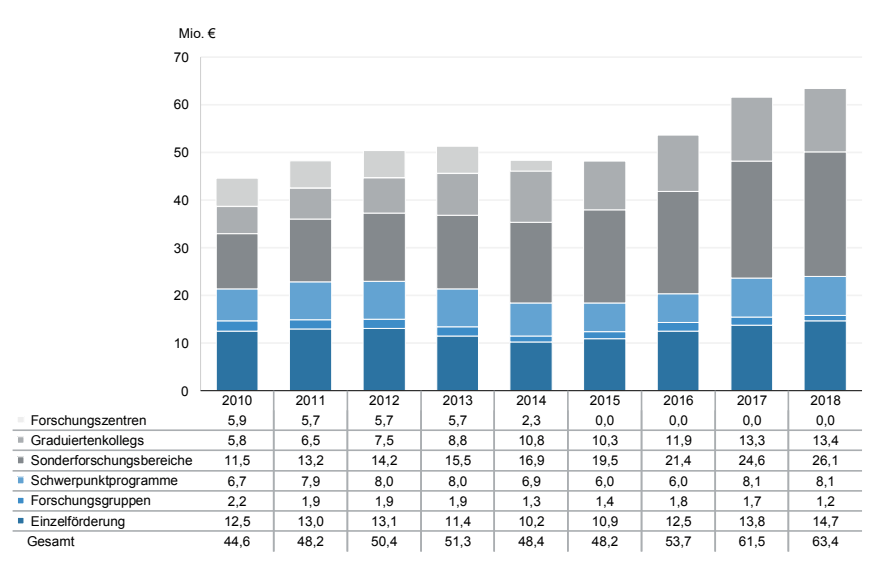

Abbildung 2. Entwicklung des Bewilligungsvolumens (Fachkollegium Mathematik). Jahresbezogene Bewilligungssummen für laufende Projekte je Programm (2010-2018) (Quelle: DFG-Fachkollegienreport 2019)

Einen Überblick über die Bewilligungssummen je Programm (Graduiertenkolleg, Sonderforschungsbereich, Schwerpunktproramm, Forschungsgruppe, Einzelförderung) findet man in Abbildung 2. Die Förderquote in der Einzelförderung liegt in der Mathematik etwas höher als die Förderquote der DFG insgesamt (siehe Abbildung 3), während die Bewilligungsquote in der Vergangenheit meist leicht unter der Bewilligungsquote der DFG insgesamt lag (siehe Abbildung 4), was dann allerdings erfreulicherweise zu einer Erhöhung des Mathematikbudgets führt.

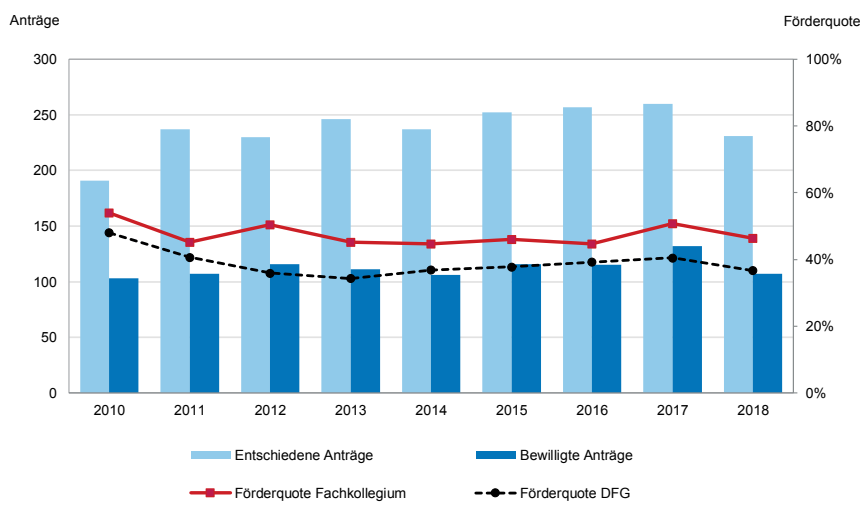

Abbildung 3. Förderquoten in der Einzelförderung (Fachkollegium Mathematik). Förderentscheidungen und Förderquoten in der Einzelförderung (2010-2018) (Quelle: DFG-Fachkollegienreport 2019) 


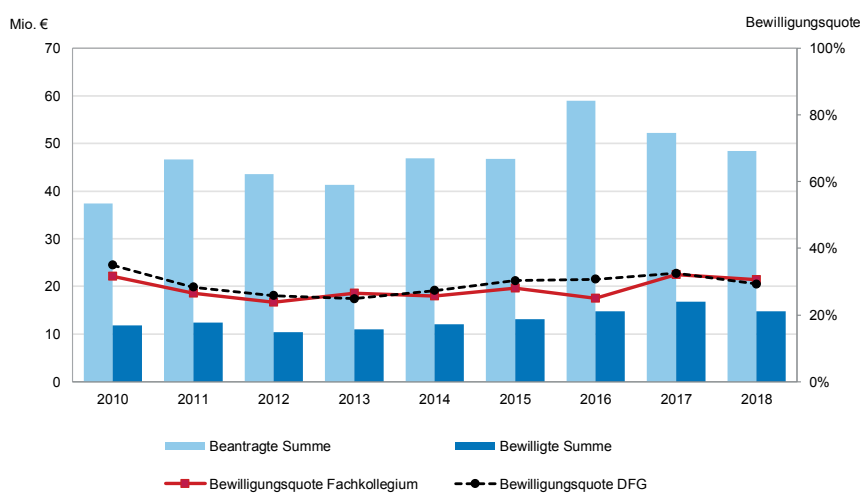

Abbildung 4. Entwicklung der Bewilligungsquoten (Fachkollegium Mathematik). Antrags- und Bewilligungssumme sowie Bewilligungsquote in der Einzelförderung (2010-2018) (Quelle: DFG-Fachkollegienreport 2019)

Dieses für die Mathematik recht positive Bild findet sich im Zusammenhang mit ERC-Anträgen leider nicht wieder. Bei ERC-Anträgen wurde festgestellt, dass seit der Gründung des ERC das Budget für Mathematik in den drei Förderbereichen (Advanced, Consolidator und Starting Grants) auf fast die Hälfte gesunken ist, da nicht genügend Anträge vorliegen. Es gibt vermutlich mehrere Gründe für diesen
Rückgang der Anzahl an Anträge, z. B. die niedrige Bewilligungsquote, das Gefühl, dass bestimmte Teilbereiche der Mathematik geringe Chancen haben, oder die Tatsache, dass es für die interdisziplinäre Erforschung der Mathematik mit anderen Wissenschaften schwierig ist, Mittel zu erhalten. Zudem wird von Antragstellenden aus der Mathematik oft beanstandet, dass das Budget, welches maximal beantragt werden kann, zu groß sei.

All dies ist teilweise richtig, aber das Nichteinreichen von Anträgen führt zu einem Teufelskreis und einem weiteren Rückgang der zur Förderfinanzierung für Projekte aus der Mathematik bereitgestellten Mittel. Dieser unglücklichen Entwicklung können wir entgegenwirken! Zum einen gibt es keinen Grund, das volle Budget zu beantragen, wenn dies nicht für ein Forschungsprojekt geeignet ist. Anträge mit kleinerem Budget sind sehr willkommen. Zum anderen sollten wir Mathematiker und Mathematikerinnen selbstbewusster sein, wenn wir Vorschläge schreiben. Es ist keine verschwendete Zeit, selbst wenn man keine Förderung erhält. Das jährliche Förderbudget für jede Disziplin wird im Verhältnis zur Anzahl der Vorschläge und dem angeforderten Budget berechnet, daher zählt jeder (gute) Antrag. Zudem führt allein die Formulierung eines guten Antrags schon sehr oft zu neuen Forschungsergebnissen.

Prof. Dr. Heike Faßbender, Technische Universität Braunschweig, Institut für Numerische Mathematik,

Universitätsplatz 2, 38106 Braunschweig

h.fassbender@tu-braunschweig.de

Prof. Dr. Volker Mehrmann, Technische Universität Berlin, Institut für Mathematik, Numerische Mathematik,

Straße des 17. Juni 136, 10623 Berlin mehrmann@math.tu-berlin.de

Heike Faßbender ist Professorin für Numerische Mathematik an der TU Braunschweig. Ihr Forschungsinteresse gilt der numerischen linearen Algebra und der Modelreduktion. Sie engagiert sich in zahlreichen Gremien, z. B. ist sie momentan Vizepräsidentin der GAMM (Gesellschaft für Angewandte Mathematik), Schatzmeisterin der ICIAM (International Council for Industrial and Applied Mathematics), Mitglied im Council von SIAM (Society for Industrial and Applied Mathematics), Mitglied im Hochschulrat der Bergischen Universität Wuppertal und Mitglied im Akkreditierungsrat.

Volker Mehrmann ist Professor für Numerische Mathematik an der TU Berlin. Seine Forschungsgebiete sind Differentiell-Algebraische Gleichungen, Kontrolltheorie, Numerische Lineare Algebra und Mathematische Modellierung sowie die Anwendung von Mathematik in der industriellen Forschung. Er ist zur Zeit Präsident der Europäischen Mathematischen Vereinigung (EMS) und er war Mitglied im DFG Fachkollegium sowie im Mathematik- und Synergy-Panel des ERC. 